\title{
Analysis of the Principal Characteristics of the On-Board Throttle Cooling System of the IR Receiver on the Basis of a Tank with Cryogenic Fueling
}

\author{
A.I. Dovgiallo, S.O. Nekrasova, D.V. Sarmin and D.A. Uglanov*
}

Samara State Aerospace University Named After Academician S.P. Koroleva (National Research University), Samara, Moscow Highway 34, Russia

\begin{abstract}
In this paper, a new tank with cryogenic charging (liquid nitrogen) is introduced. On the basis of this tank it is proposed to create a throttle cooling system, which may have enhanced characteristics compared with a throttle cooling system based on high pressure tank. This article deals with first results of tests of a tank with cryogenic charging (liquid nitrogen), which have been made at level of pressure to $4 \mathrm{MPa}$. Also it is shown that the behavior of parameters in a tank on time corresponds to calculation model.
\end{abstract}

Keywords: Cooling efficiency, cryogenic technique, tank with cryogenic fueling, throttle cooling system.

\section{INTRODUCTION}

Currently cooling of IR-receivers, elements of optical systems and other devices installed on aircrafts is an essential requirement for many aerospace projects [1]. A cooling system may have different weight, power consumption, size, reliability, cooling efficiency and thermostatic control temperature parameters. types:

Onboard cooling systems can be divided into three main

- $\quad$ Closed-cycle thermo-mechanical cooling system;

- Disposable open-cycle cooling systems using, for example, high-pressure gas;

- $\quad$ Vortex cooling system [2].

Refrigerants that are either in subcritical or supercritical state, solidified refrigerant and liquid stored at room temperature are used in these systems.

The simplest and the least expensive disposable cooling system is the cooling system based on the Joule-Thomson effect, and using gaswhich is under high-pressure equal to 7$60 \mathrm{MPa}$.

Usage of helium, hydrogen, argon or nitrogen offer an opportunity to provide a temperature level of cooling equal from $74 \mathrm{~K}$ to $4.2 \mathrm{~K}$ when the total heat capacity of the object from 0.1 to 10 watts.

Higher cleanliness requirements are placed on working bodies of bottled throttle systems that is implemented by means of their regasification from cryogenic liquid state, after which they are filled into atankwith the help of compression or bypass from high-pressure accumulators.
Operation time of throttle cooling systems depends on the mass of the fueling and required cooling efficiency.

Cryogenic liquids are also used in space engineering in cooling systems on board of aircrafts. But for some operational requirements their application causes certain difficulties.

Thus, from the above it follows that objectively useful and relevant will be development and creation of general purpose equipment which can meetthe existing technologies of compressed gases and cryogenic liquids use.

For example, at gas compressor stations with the existing infrastructure and charging technologies it would be appropriate to combine the currently used method for tanks fuelingby compression with charging from cryogenicallyliquid state.

\section{A TANK WITH CRYOGENIC CHARGING}

An example of a combination of existing and perspective technologies is a patent "Fuel Tank" of Samara State Aerospace University (patent No.2163699, Russia, IPC 7F17C9/02, 99114577/06 appl. 02.07.1999, publ. 27.02. 2001. Bul. No. 6). Fig. (1) is a sketch of the tank described in this patent [3].

Its feature is a cryogenic fueling which allows to increase the operating time of the throttle system by 2-2,5 times with the same volume and weight of charged working fluid.

The underlying ideas of this invention are that in case of recharging of the tank by a gas product, it works as a usual one, and in case of refueling it by a cryogenic component having equal massthe tank operates in more favorable pressure conditions. In addition, the internal "thermos" container for the cryogenic component and thermal insulation prevents heat strokes typical for usual tanks andmoderate the conditions of thermal cycle reliability of the structure. Calculations show that the cycles of strength load of the tank fueled using pressure are three times lower than when it is charged using compression [4]. 


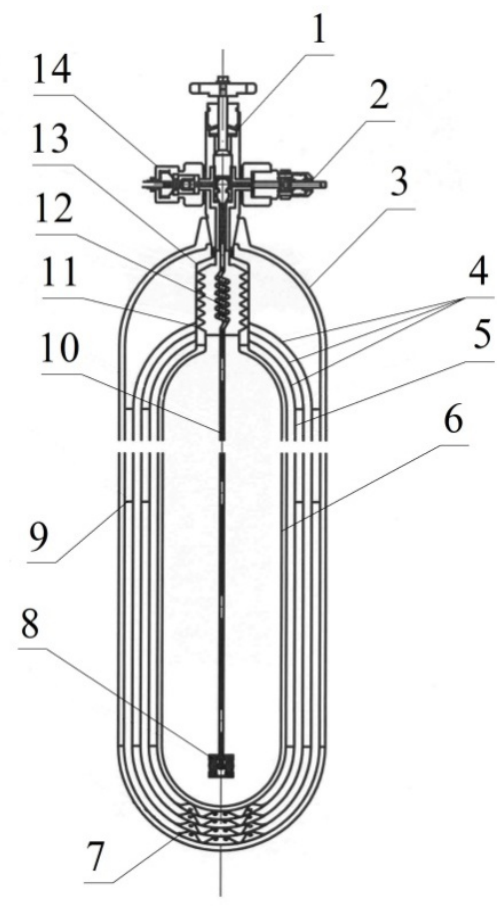

Fig. (1). 1-Tank with cryogenic fueling: 1-shut-off valve; 2-safety valve; 3-tank; 4-insulation; 5-isolation of the inner container; 6internal container; 7-bearing spacers; 8-filter; 9-radial spacers; 10tumbler; 11-filling tube; 12-coil; 13-bellow valve; 14-fitting.

\section{THROTTLE COOLING SYSTEM BASED ON TANK WITH CRYOGENIC CHARGING}

Use of this tank with cryogenic fueling in throttling cooling systems is perspective [3]. In the paper [5] the calculation of the basic characteristics of the throttle cooling systems (TCS) based on a high-pressure tank (HPT) and on the basis of a tank with cryogenic fueling (TCF). In the first case a high-pressure tank charged with nitrogen with initial pressure $p_{0}=30 \mathrm{MPa}$ and temperature $T_{0}=293 \mathrm{~K}$ is used in the throttle system.

In the second case a tank with cryogenic fueling (TCF) is used as a part of the throttle system. The made thermodynamic calculations showed that in the case of such tank fueling with cryogenic product (nitrogen) the equilibrium state of the system tank-working fluid take place in 30-40 hours. The temperature of the latter is $T_{0}=130-170 \mathrm{~K}$ at pressure $p_{0}=3 \mathrm{MPa}$. In both cases the initial mass of the working fluid is the same. Fig. (2) shows the working cycles for the two variants of the throttle cooling system.

\section{SIMULATION TESTS OF A TANK WITH CRYOGENIC CHARGING FOR THROTTLE COOLING SYSTEM}

Simulation tests of a tank with cryogenic charging for throttle cooling system showed the validity of theoretical models and calculation methods, and the reliability of the system $[6,7]$.

The tests were carried out for two modes: non-flow and charging. During the tests pressure and temperature of the tank wall, the thermos container wall, gas and liquid were recorded. Tank pressure varied from 0,1 to $1,6 \mathrm{MPa}$.
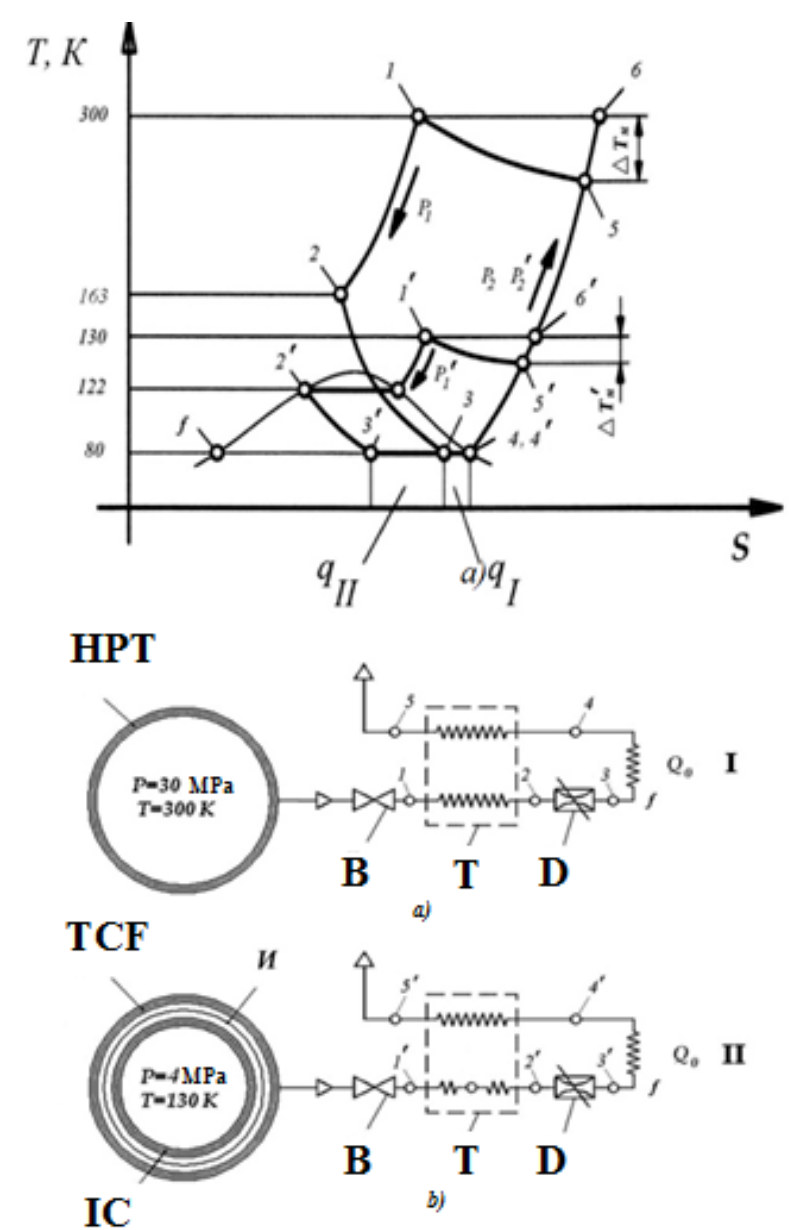

Fig. (2). Throttle cooling systems operation: a) operating process in T-S diagram; b)-throttle cooling systems diagrams: I-standard tank system; II-system TCF; HPT-high-pressure tank; B-shut-off valve; D-throttle valve; IC-inner container (thermos); I-heat insulation; TCF-tank with cryogenic fueling, T-heat exchanger.

Pressure level during the tests was limited to safety requirements, however it did not influence the thermophysical processes. Weight of the container with safety automation was $8.8 \mathrm{~kg}$ (Fig. 3). Thank wall temperature in different sections depending on the time of operationin the charging mode was recorded by thermal camera and is shown in Fig. (4).
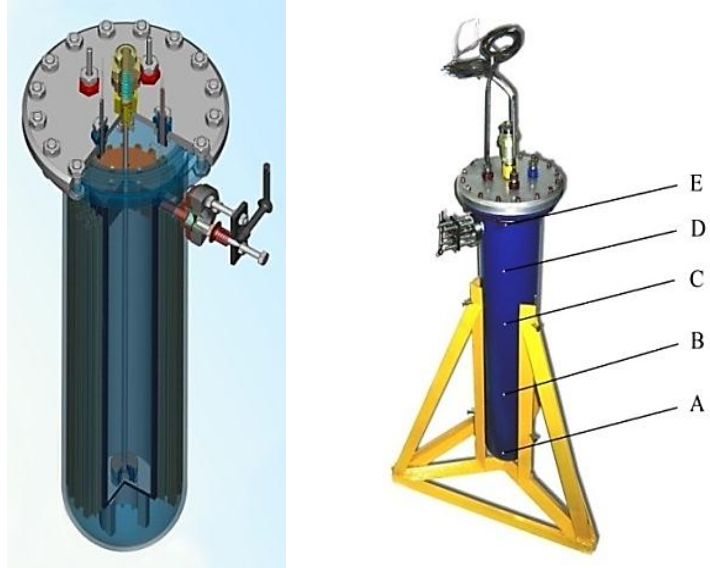

Fig. (3). Structure and photo of the external view of the TCF breadboard installation. 


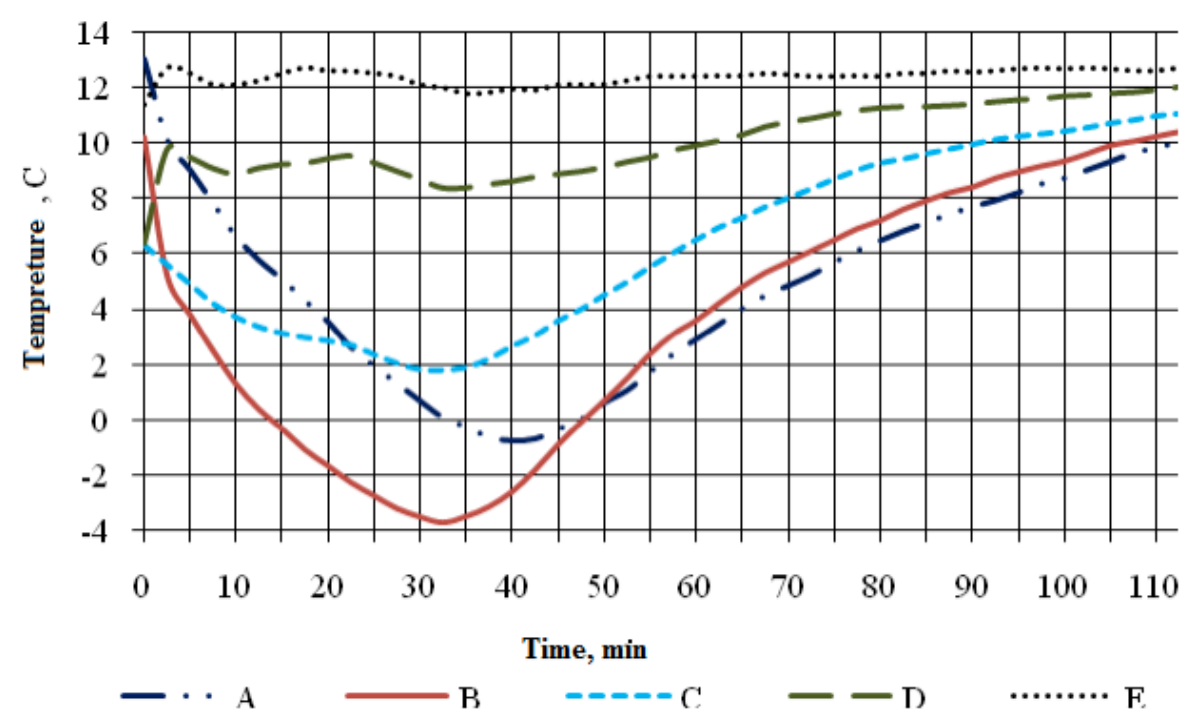

Fig. (4). Temperature distribution of the tank wall in different sections, depending on the operation time in the charging mode (points indexing corresponds to Fig. 3).

In Figs. $(\mathbf{5}, \mathbf{6})$ the experimental curves of temperature and pressure are laid down over the pressure and temperature alignment charts, obtained using the abovementioned procedure at fill pressure of $0.1 \mathrm{MPa}$ of cryogenic liquid.

Maximum misalignment of the theoretical and experimental data was $8 \%$ for the pressure and $3 \%$ for the temperature, which indicates the applicability of the techniques.

\section{TECHNICAL AND ECONOMIC ASSESSMENT OF TANK WITH CRYOGENIC CHARGING APPLICATION}

Technical and economic assessment of TCCapplication as a part of a throttle cooling system comparing to HPT shows double reduction of the system weight or proportional increase of the time of its operation compared to existing standard systems [8].

At the same time, we compared two variants of TCC as part of the TCS:

1. Cooling efficiency of TCS $\mathrm{Q}=10 \mathrm{~W}$, high-pressure tank volume and TCC is 11 .

2. Cooling efficiency of TCS $\mathrm{Q}=10 \mathrm{~W}$, high-pressure tank volume and TCC is 81 .

Analysis of the chart (Fig. 7) shows that TCS operation time can be increased by $50-80 \%$ with the help of TCC (depending on the working fluid charging mass), at the same time the mass of TCC is greater by $5-20 \%$ than the mass of TCS at equal cooling efficiency depending on the volume of tanks.

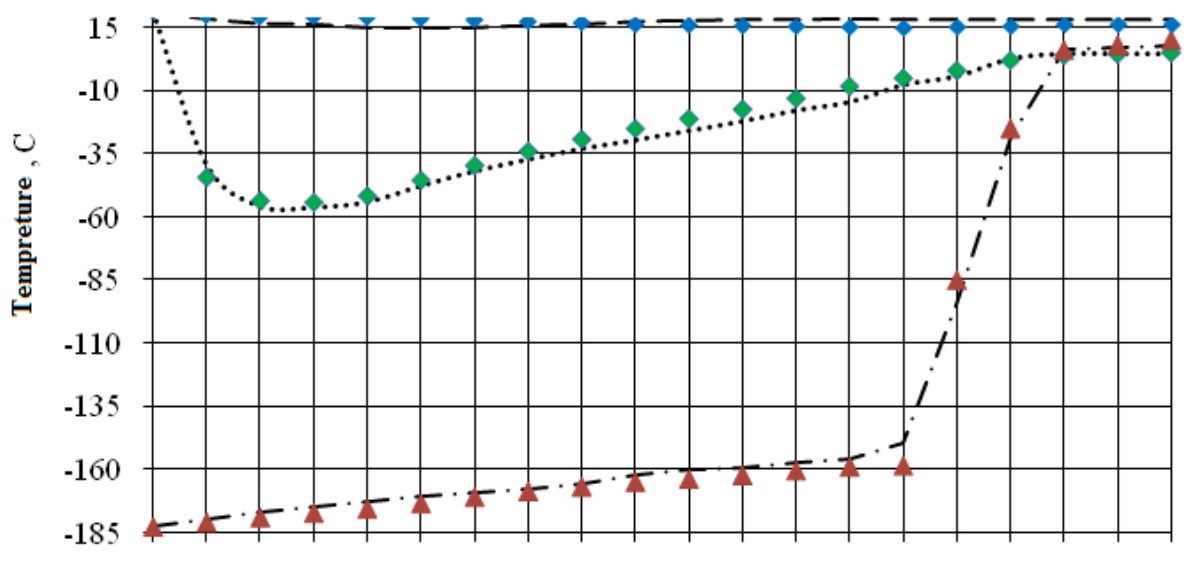

$\begin{array}{llllllllllllllllllll}0 & 3 & 5 & 8 & 10 & 13 & 15 & 19 & 22 & 25 & 28 & 30 & 35 & 38 & 41 & 44 & 47 & 50 & 52 & 55\end{array}$

Time, $\min$
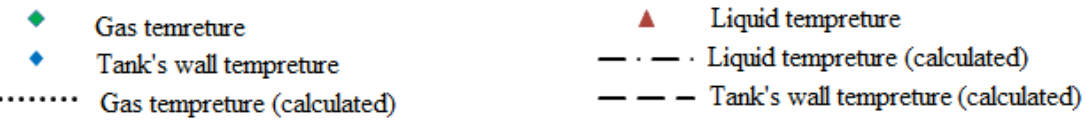

Fig. (5). Design and experimental charts of fluid, insulation, gas and TCC wall temperature changes as a function of cryogenic product storage time (non-flow mode). 


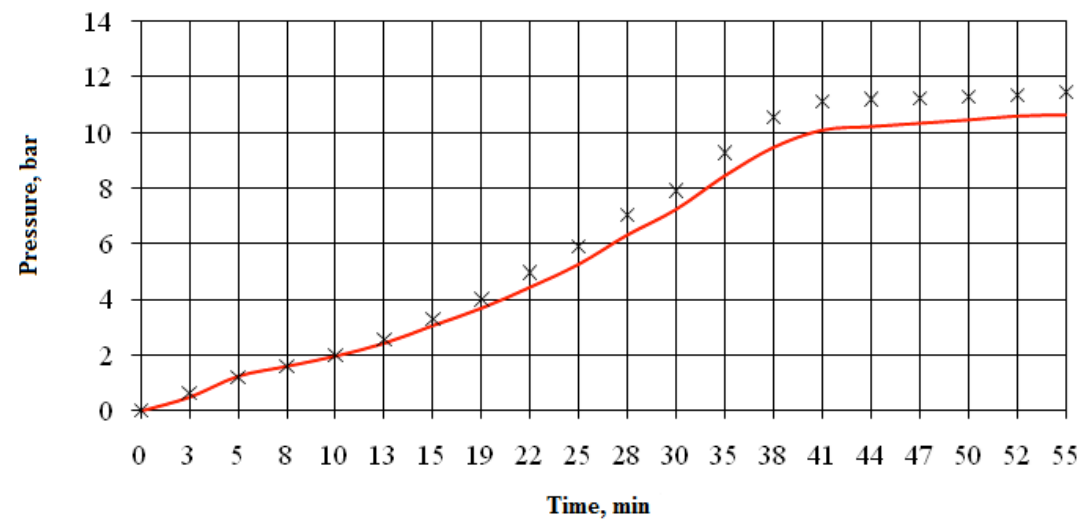

* Experiment

Calculation

Fig. (6). Design and experimental change of gas pressure as a function of the cryogenic product storage time in TCC (non-flow mode).

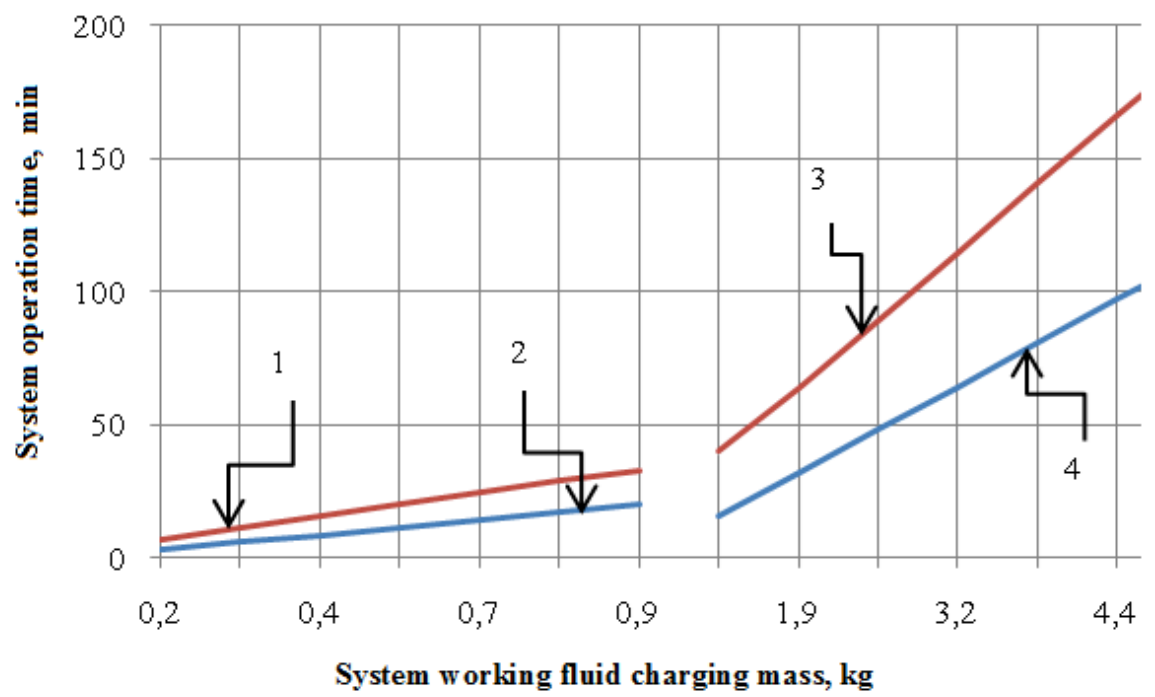

Fig. (7). Graph of variance of system operation as a function of working fluid charging mass: 1-system with TCF, tank volume-1 1.; 2-system with HPT, tank volume-1 1. 3-system with TCF, tank volume-81.; 4-system with HPT, tank volume-8 1.

To obtain an objective assessment of the effectiveness of HTP application as a part of TCS a specific value such as the operation time coefficient was introduced.

$$
\gamma=\frac{\tau_{\partial c o}}{m_{\text {бал }}}
$$

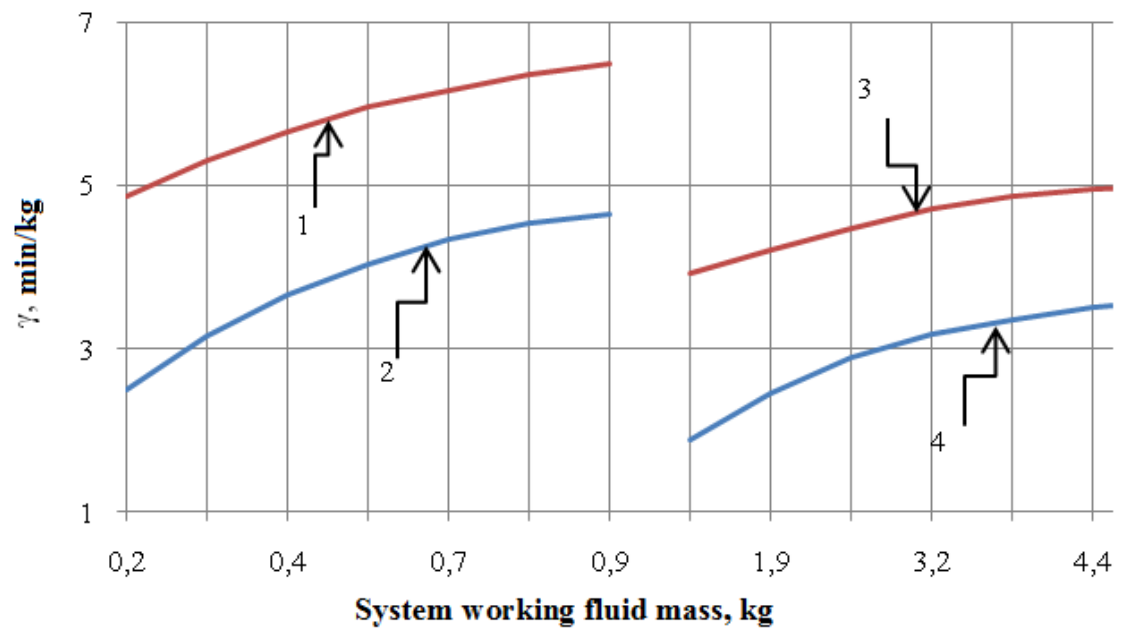

Fig. (8). Dependence of the operation time coefficient on the mass of tank charging with working fluid. 1-system with TCF, tank volume-1 1.; 2-system with HPT, tank volume-1 1. 3-system with TCC, tank volume-81.; 4-system with HPT, tank volume-8 1. 
where $\tau_{\partial с о}$ isthe throttle system operation time, and $m_{\text {бал }}$ is the working fluid mass in the system tank

Analysis of the graph (Fig. 8) shows that in case of the same charging mass TCC as a part of TCS increases the onboard cooling system operation time by a factor of 1.2-1.5 compared with HPT. Or for some fixed operation time the on-board TCS, which includes TCC, will be charged by the working fluid the quantity of which is by $40-60 \%$ less compared with TCS based on a high-pressure tank.

Based on the above it can be concluded that the use of TCF allows to reduce mass-dimensional characteristics of the cooling system and to increase the efficiency of cryogenic systems application on board aircrafts.

\section{CONCLUSION}

Thus, these indicators provide an objective assessment of the potential for additional energy when using cryogenic products with their subsequent regasification in TCF considering operational constraints and working fluid flow demand. Based on the above it can be concluded that the proposed technical solution is perspective and can be applied in various fields of technics.

\section{ABOUT THE AUTHORS}

First Author Dovgiallo Alexander Ivanovich, Professor, Ph.D. in Engineering Sciences, Academician of the International Academy of Refrigeration. Author of 65 papers and 12 patents.

Second Author Nekrasova Svetlana Olegovna, graduate student of Thermal engineering and heat engines department of SSAU. Academic Advisor of the International Academy of Refrigeration.

Third Author Sarmin Dmitry Viktorovich, teaching assistant of the of Thermal engineering and heat engines Department, Ph.D. inEngineering Sciences

Forth Author Uglanov Dmitry Aleksandrovich, Associate Professor of Thermal engineering and heat engines Department, Ph.D. inEngineering Sciences

\section{CONFLICT OF INTEREST}

The author confirms that this article content has no conflict of interest.

\section{ACKNOWLEDGEMENTS}

This work was supported by the Ministry of education and science of the Russian Federation in the framework of the implementation of the Program of increasing the competitiveness of SSAU among the world's leading scientific and educational centers for 2013-2020 years.

\section{REFERENCES}

[1] E.K. Krasnochub, "Microcryogenic spacecrafts cooling systems of infrared supervision based on microcryogenic systems", PoletSamara, vol. 11, pp. 41-8, 2004.

[2] V.V. Biryuk, "Vortex effect of gas energy separation in aircraft engineering and technology", Izvestiya Vysshikh Uchebnykh Zavedenij. Aviatsionnaya Tekhnika, 1993, voo. 2, pp. 20-23.

[3] Patent 2163699, Russian Federation, IPC 7F17C9/02/ Fuel Tank [Text] / A.I Dovgiallo, S.V. Lukachev, et al. Applicant and patentee SSAU. - No. 9911457706 appl. 02.07.1997, publ. 27.02.2001. Bull. No. 6 .

[4] A.I. Dovgiallo, and D.A Uglanov, Heat transfer and thermal-cycle strength in a fuel tank with cryogenic charging // Bulletin of SSAU, 2007, No.2.

[5] A.I. Dovgiallo, A.P. Logashkin, D.V. Sarmin, and D.A. Uglanov, Operational analysis of a bottle-stored microcooler in case of nitrogen with near-critical parameters use. Bulletin of the Samara State Aerospace University. S.P. Koroleva, No.3 (Part 2), pp. 14346, 2009.

[6] A.I. Dovgiallo, D.V. Sarmin, and D.A. Uglanov. Preliminary studies of thermal processes in a tank with cryogenic charging of the on-boardthrottle system // Bulletin of the Samara State Aerospace University. S.P. Koroleva, 2011, No.3 (Part 4), pp. 7885 .

[7] D.V. Sarmin, S.O. Nekrasova, D.A. Uglanov, and A.I. Dovgiallo, "Simulation test of a tank with cryogenic charging for a throttle cooling system and its comparative characteristics", Applied Physics, 2013, vol. 4, pp. 54-9.

[8] A.I. Dovgiallo, A.P. Logashkin, D.V. Sarmin, D.A. Uglanov, and E.S. Shatohin, "Comparison of mass characteristics of a tank with cryogenic charging and high pressure tank for throttle cooling system", Bulletin of the Samara State Aerospace University. S.P. Koroleva, Vol. 3, 112-8, 2013. 